Information

Systems and

e-Business

Management

(C) Springer-Verlag 2004

\title{
The relationship between quality of service perceived and delivered in mobile Internet communications
}

\author{
Matti Koivisto $^{1}$, Andrew Urbaczewski ${ }^{2}$ \\ ${ }^{1}$ Principal Lecturer, Mikkeli Polytechnic, Patteristonkatu 3, 50100 Mikkeli, Finland \\ (e-mail: Matti.koivisto@mikkeliamk.fi) \\ 2 Assistant Professor, University of Michigan - Dearborn, 19000 Hubbard Drive, FCS \\ 164, Dearborn, MI 48126 USA (e-mail: aurbacze@umd.umich.edu)
}

\begin{abstract}
Today mobile network operators offer a wide variety of mobile Internet services to their customers including mobile email, banking, and news services. The acceptance of these services is heavily based on quality of service (QoS) experienced by the user. So far the discussion of QoS has been very network centric and the key to user satisfaction has been the network performance.

In this paper we analyze the relationship between QoS perceived by the customer and network performance offered by the service provider. We conduct a lab experiment where network performance variables were controlled. Statistical analysis of the results indicates that there is no 1:1 correspondence or even truly linear relationship between network performance and perceived QoS as recommended by the International Telecommunications Union-Telecommunication (ITU-T). Instead the relationship varied from application to application. We examine several usability factors as potential reasons behind this phenomenon and make suggestions for future research.
\end{abstract}

Key words: quality of service, mobile Internet, network performance

\section{Introduction}

Mobile networks have undergone rapid development from the first generation analog systems to modern digital networks. The deployment of third generation systems as well as enhanced versions of second generation systems make mobile Internet and advanced mobile multimedia services available. A key factor influencing the acceptance of a new service is the balance between quality of service (QoS) perceived by users and QoS delivered by service providers. In many areas of life, perception and delivery are often different, and with QoS there is reason to expect this as well. Disappointing adoption rates among services are cause for concern and part of the motivation for studying whether there is a disconnect between QoS perceived and delivered. 
In this paper we analyze the relationship between QoS perceived by the customer and network performance offered by the service provider. We conducted trials with a sample of users and administered a satisfaction instrument, which we used to analyze the relationships between the network performance related to experienced and reported QoS. In Section 2, concepts of QoS, network performance, and usability are briefly explained. Section 3 covers QoS architectures and technologies used both in fixed and mobile networks. As Section 3 concentrates on technical aspects of QoS and network performance, Section 4 presents the framework for QoS measurements from the user's point of view. In Section 5 we describe the test design and the findings from the survey. Finally, in Section 6 we provide a few concluding thoughts and suggest directions for future research initiatives.

\section{QoS, network performance, and usability}

The phrases quality of service, network performance, and usability are extensively used today in different contexts, but the meanings of the terms are not always clear. The basic definition of QoS can be found from (ITU-T 1994), which defines QoS as: the collective effort of the service performance, which determines the degree of satisfaction to the end user. However, this is not the only (although probably the most accepted) definition. For example, the European Union's (EU) R\&D in Advanced Communications technologies in Europe (RACE) program defines QoS as (RACE 1994): "a set of user-perceivable attributes of that which makes a service what it is. It is expressed in user-understandable language and manifests itself as a number of parameters, all of which have either subjective or objective values".

According to this definition, QoS covers both subjective and objective aspects of a service. This means that the scope of QoS is a broader area than the scope of usability or network performance. Usability concerns the subjective experiences of users. The International Organization for Standardization (ISO) defines usability as the effectiveness, efficiency and satisfaction with which specified users can achieve specified goals in particular environments (ISO 1998), as recommended by other scholars such as Nielsen (Nielsen 1993). Network performance, instead, is focused on the objective performance measures of the network and it is defined as the ability of a network or network portion to provide the functions related to communication between users (ITU-T 1994).

In addition to the scope of QoS, there are also several different viewpoints from which to analyze quality. In recommendation G.1000, the International Telecommunications Union - Telecommunication (ITU-T) has presented four viewpoints of QoS (ITU-T, 2001):

- Customer's requirements of QoS

- QoS offered by the service provider

- QoS delivered by the service provider

- QoS perceived by the customer

Inter-relationships between various viewpoints of QoS, network performance and usability are shown in Fig. 1.

As shown in Fig. 1, QoS combines users and networks under the same umbrella. Part of QoS can be addressed under the auspices of usability, while 


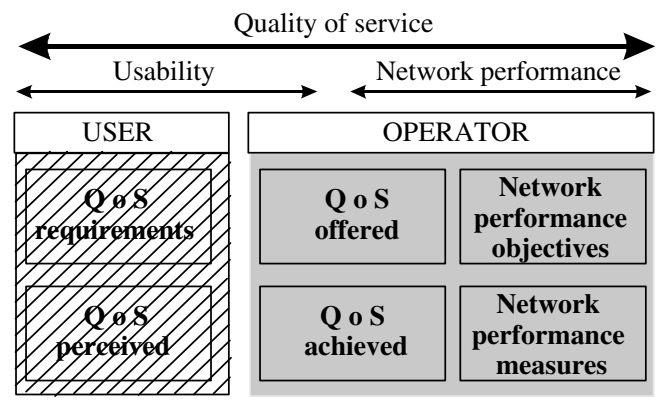

Fig. 1. Viewpoints of QoS (based on ETSI, 1994)

the rest can be more directly measured by the actual network performance. Usability is more in the eyes of the user, while the operator is concerned with the speed and reliability that the network delivers. QoS brings these two concepts together in one model. QoS has been studied intensively on factors such as bandwidth, jitter, and packet loss (see Wood and Chatterjee 2002 for a review), but has been studied only lightly from the user's point of view, as perceived QoS. The $9^{\text {th }}$ annual WWW user survey conducted by Georgia Tech, is one of those studies examining perceived QoS. It uses a survey methodology to ask users about the most important factors of QoS, and users rated mean response time as the most important QoS factor (GVU 1998), which is then taken for granted in other studies using simulations, such as da Fonseca and Oliveira (2002). In order to provide a more detailed review, in the next sections we will analyze QoS first from the network's and then from the user's side.

\section{Quality of service in fixed and mobile networks}

\subsection{QoS architectures}

IP protocols developed by the Internet Engineering Task Force (IETF) have originally been designed for fixed, best-effort networks. The use of IP in delay sensitive applications has created new challenges to QoS aspects of the network. The notion of supporting QoS over packet networks is not new, and a number of research papers, and efforts have appeared over the years to support this capability (Metz 1998).

The IETF's QoS architectures are typically classified into two categories: integrated services (IntServ) and differentiated services (DiffServ) model. The IntServ model merges the advantages of datagram and circuit-switched networks by specifying characteristics of the transmitted flow (Manner et al. 2002). It uses the resource reservation protocol to propagate the attributes of the data flow and to request specific resources along the data path (Wroclawski 1997).

While IntServ provides per-flow QoS guarantees, DiffServ maps data flows into a few priority levels - an approach sometimes referred to as Class of Service (CoS). In DiffServ there is no explicit negotiation between the application and the network about the capability to meet the QoS requirements of the application. As a result of this, DiffServ is more approximate in the nature of its outcomes but it has excellent scaling 
properties (Kan 2001). More information about IntServ and DiffServ models can be found in (Braden et. al. 1994) and (Grossman 2002).

\subsection{IP and mobility}

Mobility support for the Internet is based on the concept of Mobile IP (Perkins 2002), which allows transparent routing of IP datagrams to mobile nodes. The concept behind mobile IP is that each mobile node is always identified by its home address, regardless of its current point of attachment to the Internet. In addition to the static home address, each node is also associated with a dynamic care-of address while situated away from its home. The care-of address provides data about the node's current attachment to the Internet and the protocol provides mechanisms for registering the care-of address with a home agent. The data to the mobile station is first sent to the home address from which the home agent sends it to the care-of address through a tunnel. Once at the end of the tunnel, data is delivered to the mobile node.

\subsection{QoS in mobile networks}

QoS management in mobile networks introduces new challenges to the IP networks. According to Mocchapatra et al. (2003), the most important issues and difficulties for supporting QoS in the mobile environment include unpredictable link properties, node mobility, limited battery life, hidden and exposed terminal problems, route maintenance, and security.

The mobility of the host has an essentially significant impact on the QoS in mobile environments. While moving from one location to another, the data flow path changes. The network parameters in this new situation may be completely different from the original one.

Over the past several years there has been a considerable amount of research within the field of QoS support for mobile IP networks. These works have mainly been based on IntServ and DiffServ models developed for wired Internet. Talukdar et al. (1997) (2001), have proposed mobility independent reservation support for Resource ReSerVation Protocol (RSVP) called MRSVP. Other IntServ based suggestions for mobile networks include (Paskalis et al. 2003) and (Kuo et al. 2000). All these proposals, however, share the basic scalability problem of the IntServ model. Other problems have been highlighted (Thomas 2002).

A similar range of difficulties exists with incorporating the DiffServ model into mobile Internet QoS. Braun et al. (1999) lists several problems occurring when DiffServ is used in the mobile environment, including lack of dynamic configuration, definition and selection of service level agreements (SLAs), mobile flow identification, and billing.

The lack of a widely accepted QoS solution for mobile networks also introduces interoperability issues. Thomas (2002) questions what ought to happen when the QoS policies at one access router are different from the QoS policies at another. For example, if the first one is supporting IntServ and the second one the DiffServ model, what kind of adaptation is required? 


\section{Measuring the QoS from user's point of view}

Architectures and techniques introduced above have an effect on network performance. According to the ITU-T terminology, they are directly connected to the QoS offered and delivered by the service provider. Although network performance has an important role in the QoS experienced by the user, they are not the same construct, and they by design are measured differently. Network performance is an absolute that can be measured in terms of bits and seconds, with metrics like delay and jitter, while user perceptions are psychological constructs that must be measured indirectly using psychological instruments. Thus one must use different methods to analyze the QoS expected and perceived by users.

There are two major approaches for the measurement of QoS from the user's point of view: opinion polls and customer satisfaction surveys (Noll 1999). An opinion poll is where anyone is asked for an opinion, and customer satisfaction tests are where users report their satisfaction levels shortly after they experience a service. Opinion polls can be used for analyzing customer requirements, and customer satisfaction surveys for studying the perceived quality.

In this case, an opinion poll probably does not provide much meaning. This would be a simple survey that anyone could answer without any prior knowledge of their experience with the technologies nor an opportunity to observe them in action. Customer satisfaction tests are closely related to usability testing. Rubin (1994) defines usability testing as techniques to collect empirical data while observing representative end users using the product to perform representative tasks. The key of this definition is the product itself. The usability of the product is analyzed in a real environment and many scholars have defined efficient usability testing procedures (Faulkner 2000; Dumas et al. 1999; Nielsen 1993).

The main difference between usability testing and customer satisfaction tests is the focus. As mentioned above, in usability testing the focus is on a single product and the manufacturer wants to identify the relative level of user friendliness for their product. A customer satisfaction survey does not analyze a single product but rather quality of service in general. Although there is a difference between the focus of these two approaches, similar methods can be used for both of them in test design and data collection.

\section{Study design and results}

\subsection{Study design}

In Sect. 3 we introduced the QoS architectures used today in IP based networks. Then, in Sect. 4 we measured QoS service from the user's point of view. Little research exists on the relationship between these two constructs, and the purpose of this study is to identify what (if any) relationship is there.

According to ITU-T (2001) there would ideally be a 1:1 correspondence between delivered and perceived QoS. Our research hypothesis is that the relationship between network performance and QoS experienced by the user is not as straightforward as recommended by ITU. QoS perceived by the user 
is much more complex, affected not only by the network performance, but also by context, application type, prior experiences, etc.

In order to test our research hypothesis, we carried out a customer satisfaction survey. Subjects interacted with three different mobile Internet applications (an email application, a banking service, and a news service) in the lab environment where network performance variables (connection establishment delay, bandwidth and connection release delay) were controlled. These applications were chosen as their domain should and/or will be familiar as the "killer apps" to mobile and Internet users (Davies 2001, Jones 2001) and for their differing levels of required interactivity.

Subjects were presented with the mobile device, a Compaq iPaq 3870 PDA with IEEE 802.11(b) WLAN connections, and an information sheet detailing the tasks they would be asked to complete. The iPaq had a 64,000 color screen and the display size was approximately 2.25 inches wide $\times 3$ inches tall. The virtual keyboard and stylus was briefly demonstrated to the students so that they would know how to enter information. Once the subjects were handed the device and signalled they were ready to begin, the subjects came to a blank "logon" screen, where the students inputted the phrase "READY1" to begin the session. This was done primarily to ensure that they were familiar with the keyboard and input process.

In the email application, the subjects were first required to "login" to an account with the username and password provided to them by the facilitator. Then each subject typed a message with the recipient, subject, and message all stipulated for them by the facilitator. In the news application (see Fig. 2 below), subjects were required to scan through the news service to find information on a particular story and then write that information down on a response sheet. In the banking application, subjects again "logged in" to a bank account with a username and password provided to them by the

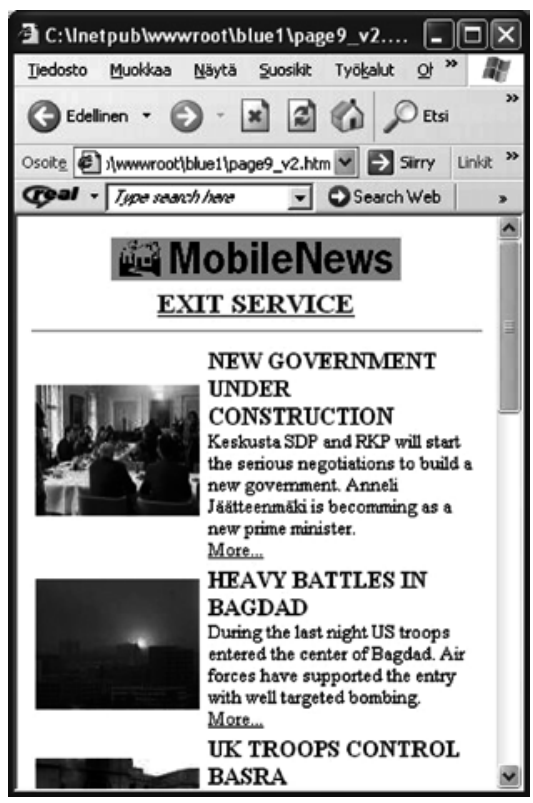

Fig. 2. Sample of the news application created for the study 


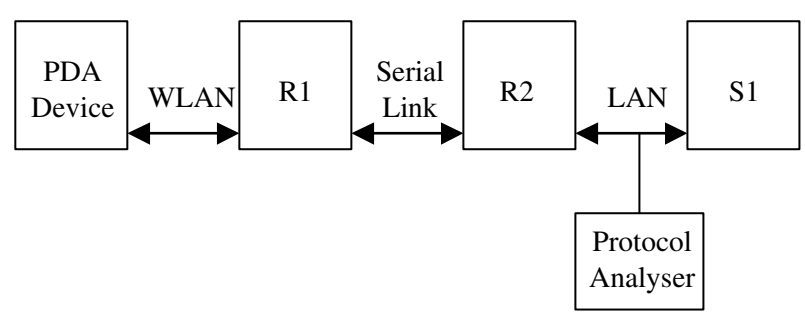

Fig. 3. Test environment

facilitator. They then paid a bill by transferring money from one account to another person's account (all information provided by the facilitator) in a means common to bill-paying applications available through the Web today. These directions were similar to other studies involving mobile device text input (e.g., James and Reichsel 2001). All of the applications had a combination of text and graphics included in the presentation, but they were low-resolution graphics typical for mobile devices.

Also, these applications were all created by the researchers but appeared to the subjects as if they were actually coming from the Internet. This was done to tightly control the application response times and the actual traffic flow over the network while simulating a real-world Internet experience. Our test environment is shown in Fig. 3.

The bandwidth was controlled by a serial line connection between two routers (R1, R2) and connection establishment and release delays were controlled in the server (S1). The protocol analyzer was then used for logging data about the study should anything appear to be not functioning correctly.

Three logical network performance levels (low, medium and high) were used and the delay and bandwidth parameters of the levels are shown in Table 1. Subjects were provided with direct clues from the screen such that they were not wondering what might be causing a certain delay. The screen would turn all white except for the text "Opening connection please wait" or "Closing connection please wait".

Subjects evaluated the services with the Absolute Category Rating method (ITU-T 1999; Noll, 1999). The method specifies that after each task users are asked to evaluate the quality of the service on a scale of 1 to 5 and the voting time is limited to 10 seconds. Subjects were not informed of the speed level they were using (in absolute or relative terms), nor were they cognizant that connections were somehow being manipulated by the researchers. We used the Latin square ordering technique in assigning subjects and cases to prevent any systematic distortions in the data due to a learning effect.

Table 1. Network performance levels used in the experiment

\begin{tabular}{lrll}
\hline Level & Bandwidth & $\begin{array}{l}\text { Connection } \\
\text { establishment } \\
\text { delay }\end{array}$ & $\begin{array}{l}\text { Connection } \\
\text { release delay }\end{array}$ \\
\hline Low & $9,600 \mathrm{bit} / \mathrm{s}$ & 6 seconds & 6 seconds \\
Medium & $56,000 \mathrm{bit} / \mathrm{s}$ & 3 seconds & 3 seconds \\
High & $256,000 \mathrm{bit} / \mathrm{s}$ & 1 seconds & 1 seconds \\
\hline
\end{tabular}


Table 2. Subject demographic information

Average usage of Internet

Average usage of mobile phones

Have used SMS service

Have used MMS service

Have used GPRS

Have used mobile email

Have used PDA devices

5.7 years
4.9 years
$95 \%$
$14 \%$
$18 \%$
$5 \%$
$4 \%$

5.7 years

$95 \%$

$14 \%$

$5 \%$

We used 142 subjects (100 males and 42 females, $89.4 \%$ between 16 and 25 years old) from the university, polytechnic and vocational school undergraduate population. All of the subjects had used both mobile phones and Internet for several years. Basic information about the test users is shown in Table 2.

\subsection{Results}

Subjects were asked to respond to a variety of questions about their experience. These questions were drawn from the ITU literature on the dimensions of QoS, and they directly related to the variables that were being directly manipulated.

We then tested these questions to ensure that we were measuring the same construct, in this case Quality of Service. We ran a factor analysis for each of the three applications, and each returned a single factor model with no second eigenvalue greater than one. The amount of variance explained was $70.5 \%, 67.1 \%$, and $69.9 \%$ for the email, news, and bank applications, respectively.

We then tested to see if the means for the treatment groups were unequal. To do this, we ran simple tests to calculate confidence intervals for the perceived QoS means in each group. We then report the descriptive statistics of our study reported in Tables 5 through 7 in the Appendix and shown graphically in Fig. 4 below.

The $\mathrm{F}$ and $\mathrm{p}$ scores reported in Tables 5 through 7 (see Appendix) show that indeed we cannot assume equal means for the perceived QoS scores in any of the three applications, which shows that there is a significant relationship between QoS delivered and perceived at the $\mathrm{p}<.05$ level. However, the data also show the difference in perceived QoS among the different levels, but not consistently for each application nor between all levels. In the banking application $(\mathrm{F}(2,139)=7.936, \mathrm{p}<.001)$, perceived QoS is highest at the high speed and significantly different than the medium

Table 3. Questions related to the network performance

Q1

Q2

Q3

Q4
Evaluate Quality of Service in Connection Establishment Phase

Evaluate Quality of Service in Data Transfer Phase

Evaluate Quality of Service in Connection Release Phase

Evaluate General Quality of Service 
Table 4. Check for between-subjects effects on perceived QoS $\left(r^{2}=.139\right.$, adj. $\left.r^{2}=.122\right)$

\begin{tabular}{lcrrr}
\hline Source & $\begin{array}{l}\text { Type III sum } \\
\text { of squares }\end{array}$ & df & $\begin{array}{l}\text { Mean } \\
\text { square }\end{array}$ & F \\
\hline Corrected model & 21.824 & 8 & 2.728 & $8.409 * * *$ \\
Intercept & 7890.233 & 1 & 7890.233 & $24322.100^{* * *}$ \\
Characters typed & 2.645 & 2 & 1.322 & $4.076^{* *}$ \\
Bandwidth & 13.326 & 2 & 6.663 & $20.539^{* * *}$ \\
Char typed * bandwidth & 5.940 & 4 & 1.485 & $4.577^{* * *}$ \\
Error & 135.277 & 417 & .324 & \\
Total & 8049.903 & 426 & & \\
Corrected total & 157.101 & 425 & & \\
\hline
\end{tabular}

speed, but perceived QoS at the medium and low speeds are statistically identical. In the email application $(\mathrm{F}(2,139)=3.499, \mathrm{p}<.033)$, there is no significant separation between the groups. While the medium speed is close to being significantly different from the low speed, at the higher speed the nominal perceived QoS seems to be lower than the medium speed. In the news application $(\mathrm{F}(2,139)=18.932, \mathrm{p}<.000)$, the perceived $\mathrm{QoS}$ at the medium speed is much higher than at the lowest speed, but perceived QoS scores are almost identical at the medium and high levels.

Several covariates were collected to see if they could help explain the data set. These variables included gender and previous experience with Short Message Service (SMS), Multimedia Messaging Service (MMS), mobile phones (GSM), Wireless Application Protocol (WAP), General Packet Radio Services (GPRS), personal digital assistants (PDA), and mobile email technologies. Univariate Analysis of Variance (ANOVA) models were then run with the perceived QoS for each application as the dependent variable, but this time included several potential covariates. Few of these potential covariates were found to be significant:

As can be seen from Tables 8 through 10 (see Appendix), there is a statistically significant difference between perceived and delivered QoS in the three applications. With news service $\left(r^{2}=.300\right.$, adjusted $\left.r^{2}=.246\right)$ and mobile banking $\left(\mathrm{r}^{2}=.182\right.$, adjusted $\left.\mathrm{r}^{2}=.119\right)$, the quality of service perceived was much more sensitive to the changes in network performance, though in the e-mail application $\left(\mathrm{r}^{2}=.127\right)$, adjusted $\mathrm{r}^{2}=.060$ the $\mathrm{p}<.054$ approaches commonly accepted levels of significance testing. Prior use of GPRS technologies seems to be a significant covariate in determining perceived QoS, though not in the news application. No specific theoretical basis exists for believing many of the potential covariates could be significant, but it makes sense that a user familiar with the technologies might be more sensitive to perceived QoS differences.

\subsection{Discussion}

Two main results of our study were:

- Users cannot separate network performance into multiple factors like connection establishment time, bandwidth, and release time but they consider it as a single factor. 


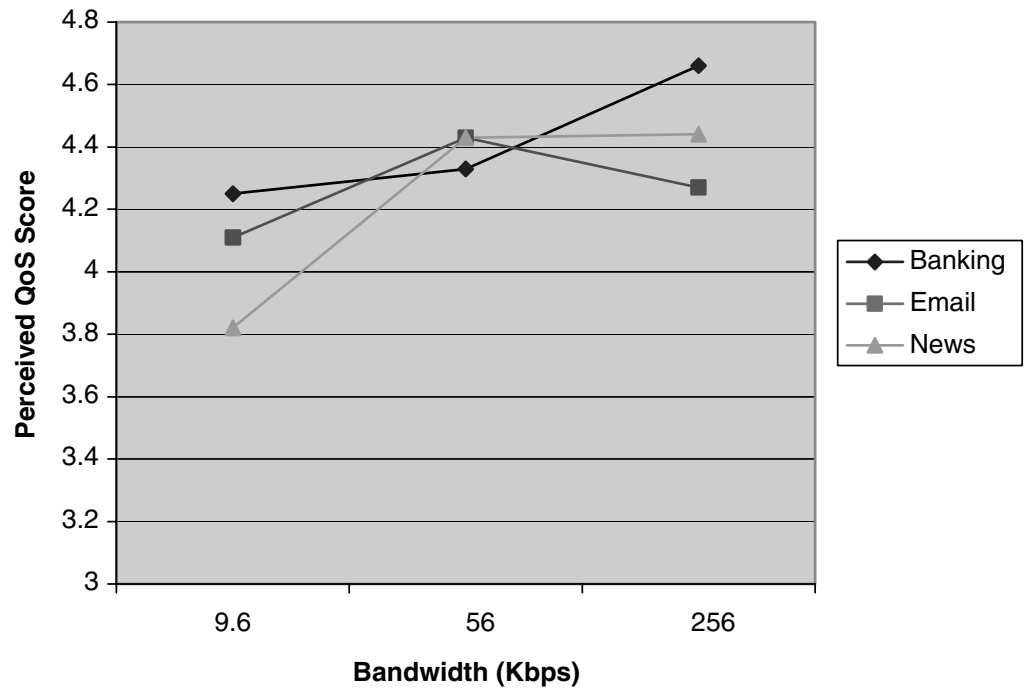

Fig. 4. QoS perceived and delivered for the three applications

- The relationship between network performance and QoS perceived is application specific.

The first finding is based on 1) about $60 \%$ of subjects gave the same value to all four questions related to the network performance (see Table 3, and 2) the strength of the factor analysis. They were not able to detect any difference between the effect of connection establishment delay, bandwidth, and connection release delay to the general quality of the service.

One of the aims of this study was to identify the relationship between different aspects of network performance and perceived QoS. We used three network performance metrics (connection establishment delay, bandwidth and connection release delay), which combined to make one perceived QoS factor.

What can explain these findings? Engineers use network performance related terms like delay, jitter, bandwidth and packet loss when considering QoS. Users do not share the network model of the engineers, and they define the quality with reference to a particular user activity (Bouch et. al. 2001a). Perceived QoS may depend on many factors, such as the screen size and processor speed, which were controlled, but may also depend on input capabilities as tested below.

We did not find the direct linear relationship between delivered and perceived QoS that was suggested by ITU. Rather, we found a general upward trend, but the users' definition and expectation of QoS depends on what their goals. The acceptable network performance level is different across applications.

This leads us to a new question - why does perceived QoS vary with the application being completed? One alternative could be found in the stress and discomfort created by the application to the user (see Bouch et. al., 2001b). Our study indicated that perceived QoS for the email application did not correlate with the network performance, unlike it did in the cases of mobile banking and news service. If the stress level is accepted as an indicator 
of perceived QoS there must have been some other and stronger source of stress than network delay in email service.

One good candidate for a source of stress is input method or typing of the message. The amount of characters typed in each tested application varied from 0 (news) to 47 (banking) to 110 (email). Bearing in mind that $96 \%$ of the subjects have never before used PDA devices with touch screens it is very likely that the subjects found it stressful to type the message with the stylus pen.

While it was not a focus of this research, the stress that played a role is likely due to the applications involved. Earlier we quote Nielsen and the ISO as defining usability in terms of effectiveness, efficiency, and satisfaction for achieving the goals in specified environments. QoS deals with the efficiency of the application's network interaction, but there are other factors at work, such as the number of characters typed and the interface. To check for interaction effects, we pooled the test results into 426 observations (142 subjects each completing 3 applications) and checked their perceived QoS scores (dependent variable) against the QoS delivered and the number of characters. The results are shown in Table 4.

While there are main effects for both characters typed and bandwidth, the interaction effect is most telling in the subjects' definition of perceived QoS. Subjects apparently are unable or unwilling to recognize the quality of the network separately from the quality of the application. Along with stress, it is possible that if the subjects were spending more time directly interacting with the application, especially as the tasks grow in labor-intensity, it could be that the subjects are not able to focus on QoS delivered, nor do they care as much about the actual performance metrics. Though we could look at any one of the applications that the subjects interacted with separately and try to make claims from that data, it is obvious that the application itself plays a large role in perceived QoS. Further research is necessary to isolate network effects from application effects.

\subsection{Limitations}

As with all research studies, there are several limitations to our work. This study was conducted under laboratory settings, where we maximized precision at the expense of realism and generalizability (McGrath 1982). Secondly, the usual caveats about student subjects may be appropriate, but in this case, young people and students are often the biggest users of this technology so we feel the subject pool is appropriate.

In this study, it is also possible that there are conflicts between the delivery of text (generally low-bandwidth) and graphics (potentially high-bandwidth) in the applications. We made no attempt to alter each individual service between low and high-bandwidth solutions, rather presenting the viewer with one option for each of the e-mail, banking, or news applications (see Fig. 2 earlier in the paper for a sample screenshot). This could be a potential confound.

It is important to note that only one type of mobile device, the compaqipaq, was used for testing in this program. It is quite possible that devices with different operating systems (such as Palm or Symbian) or other interfaces (e.g., 12-key, Graffiti) might have produced different results. It is 
also possible that the delays chosen for connection establishment and release were not long enough to create perceived differences, as they were chosen using only common examples of short, medium, and fast page loading times.

Moreover, it is possible that there are some characteristics of this experiment that are not extendable to all mobile applications. While we chose three reasonably diverse applications, they were all static. It is possible that applications which use real-time continual interaction, such as with streaming media or online gaming, might produce different results. This is an area for future study.

\section{Conclusions}

The acceptance of mobile Internet services is heavily based on quality of service experienced by the user. So far the discussion of QoS has been very network centric and the key to user satisfaction has been the network performance.

In our user satisfaction survey we discovered that users do not model the effect of network performance to QoS in the network centric terms like delay and bandwidth. The second finding of our study was that there is no $1: 1$ correspondence between network performance and perceived QoS as recommended by the ITU-T. The relationship varied from application to application. A possible reason for these differences between applications can be the concept of user cost. User cost refers here to the stress and discomfort resultant from the usage of the service. However, we need to carefully state that this idea requires further study.

Network providers may wish to examine the types of applications that their users demand (and actually use) in relationship to the networks that they currently provide. Billions of dollars were spent around the world on mobile 3G licenses that are largely going unused. While there are certainly many factors involved in the telecom collapse of the early 21 st century, one cannot ignore that the supply has not created its own demand, nor the willingness on the part of the users to upgrade their devices to take advantage of applications which they may not use or may not have a large enough network of common users to participate. Rather, if users will continue with the same applications, the same old network will suffice. Network providers should focus on both supply and demand in making rollout decisions.

\section{References}

Bouch A, Sasse M (2001a) Designing QoS and Charging Mechanisms: He Who Pays the Service Shapes the Design. Proceedings of ICQT'01. Vienna, Austria

Bouch A, Wilson G, Sasse M (2001b) A 3-Dimensional Approach to Assessing End-User Quality of Service. Proceedings of the London Communications Symposium, pp 47-50

Braden R, Clark D, Shenker S (1994) Integrated Services in the Internet Architecture: an Overview. Internet Engineering Task Force, Request for Comments (RFC) 1633

Braun T, Castelluccia C, Stattenberger G (1999) An Analysis of the DiffServ Approach in Mobile Environments. IQWiM-Workshop'99

Davies W (2001) Wireless office on the move. International Telecommunications, 35(10): 33-38

Dumas J, Redish J (1999) A Practical Guide to Usability Testing. Revised Ed. Intellect, Exeter 
ETSI (1994) General Aspects of Quality of Service and Network Performance, European Telecommunications Standards Institute. Technical report ETR 003

Faulkener X (2000) Usability Engineering. Palgrave, New York

Fonseca NLS da, Oliveira, RM (2002) Role of Download Time as a Key in Web Cache Management Policies. Journal of Network and Systems Management, 10(1): 1-6

Grossman D (2002) New Terminology and Clarifications for Diffserv. Internet Engineering Task Force, Request for Comments (RFC) 3260

GVU (1998) Graphics, Visualization, and Usability Center, Georgia Tech, $9^{\text {th }}$ Web User Survey, http://www.gvu.gatech.edu/user_surveys/survey-1998-04/

ISO (1998) Ergonomic Requirements for Office Work with Visual Display Terminals (VDTs) Part 11: Guidance on Usability. International Organisation for Standardization. 9241-11

ITU-T (1994) Terms and Definitions Related to Quality of Service and Network Performance including Dependability. Recommendation E.800

ITU-T (1999) Subjective Video Quality Assessment Methods for Multimedia Applications. Recommendation P.910

ITU-T (2001) Communications Quality of Service: A Framework and Definitions. Recommendation 6.1000

James CL, Reichsel KM (2001) Text Input for Mobile Devices: Comparing Model Prediction to Actual Performance, ACM SIGHCI 2001 Conference Proceedings, pp 365-371

Jones S. (2001) M-Banking the Killer Application. Business Communications Review International 1(7): 18-20

Kan Z, Zhang D, Zhang R, Ma J (2001) QoS in Mobile IPv6. Proceedings of Int. Conference on Information Technology and Information Networks, vol. B, pp 492-497, Beijing, China

Kuo G, Ko P (2000) Dynamic RSVP for Mobile IPv6 in wireless networks, IEEE Vehicular Technology Conference, Tokyo, Japan

Manner J, Lopez A, Mihailovic A, Velayos H, Hepworth E, Khouaja Y (2002) Evaluation of Mobility and QoS Interaction. Computer Networks, 38(2): 137-163

McGrath JE (1982) Dilemmatics: The Study of Research Choices and Dilemmas In: McGrath JE (ed.) Judgement Calls in Research, Beverly Hills: Sage, pp 69-80

Metz C (1998) IP Switching - Protocols and Architectures. McGraw-Hill. London

Mohapatra P, Li J, Gui C (2003) QoS in Mobile Ad hoc Networks. Special Issue on QoS in Next-Generation Wireless Multimedia Communications Systems. IEEE Wireless Communications Magazine 10(3)

Nielsen J (1993) Usability Engineering. Academic Press, San Diego

Noll J (ed) (1999) Quality of Service (QoS) Measures for Applications, EURESCOM Project P921

Paskalis S, Kaloxylos A, Zervas E, Merakos L (2003). An Efficient RSVP-mobile IP Interworking Scheme. Mobile Networks and Applications 8(3):197-207

Perkins C (2002). IP Mobility Support for IPv4. Internet Engineering Task Force, Request for Comments ( RFC) 3344

RACE (1994) UMTS System Structure Document, Issue 1.0. RACE 2066 Mobile Networks (MONET), CEC Deliverable No:R2066/LMF/GA1/DS/P/052/b1

Rubin J (1994) Handbook of Usability Testing John Wiley \& Sons, New York

Talukdar A, Badrinath B (1997) IPv6 + Mobile-IP + MRSVP = Internet Cellular Phone? Proceedings of the International Workshop On Quality of Service, Columbia University, New York, USA

Talukdar A, Badrinath B, Acharya A (2001) MRSVP: A Resource Reservation Protocol for an Integrated Services Network with Mobile Hosts. Wireless Networks, 7(1)

Thomas M (2002) Analysis of Mobile IP and RSVP Interactions. Internet Engineering Task Force, Internet Draft

Wood S, Chatterjee S (2002) Network Quality of Service for the Enterprise: A Broad Overview. Information Systems Frontiers, 4(1): 63-84

Wroclawski J (1997) The Use of RSVP with IETF Integrated Services. Internet Engineering Task Force, Request for Comments (RFC) 2210 


\section{Appendix: Stastical test results}

Table 5. Descriptives for perceived QoS differences between groups with the Banking app.

\begin{tabular}{lclllll}
\hline $\begin{array}{l}\text { Connect } \\
\text { Speed }\end{array}$ & $\mathrm{N}$ & $\begin{array}{l}\text { Bank app } \\
\text { QoS Mean }\end{array}$ & SD & SE & $\begin{array}{l}95 \% \text { CI } \\
\text { lower bound }\end{array}$ & $\begin{array}{l}95 \% \text { CI } \\
\text { upper bound }\end{array}$ \\
\hline Low & 48 & 4.2448 & .58911 & .08053 & 4.0737 & 4.4159 \\
Medium & 47 & 4.3298 & .62794 & .09159 & 4.1454 & 4.5142 \\
High & 47 & 4.6649 & .37328 & .05445 & 4.5553 & 4.7745 \\
Total & 142 & 4.4120 & .56822 & .04768 & 4.3177 & 4.5062 \\
\hline
\end{tabular}

Table 6. Descriptives for perceived QoS differences between groups with the Email app.

\begin{tabular}{lrlllll}
\hline $\begin{array}{l}\text { Connect } \\
\text { speed }\end{array}$ & $\mathrm{N}$ & $\begin{array}{l}\text { Email app } \\
\text { QoS mean }\end{array}$ & SD & SE & $\begin{array}{l}95 \% \mathrm{CI} \\
\text { lower bound }\end{array}$ & $\begin{array}{l}95 \% \mathrm{CI} \\
\text { upper bound }\end{array}$ \\
\hline Low & 47 & 4.1064 & .71806 & .10474 & 3.8956 & 4.3172 \\
Medium & 48 & 4.4323 & .45762 & .06605 & 4.2994 & 4.5652 \\
High & 47 & 4.2660 & .59983 & .08749 & 4.0898 & 4.4421 \\
Total & 142 & 4.2694 & .61098 & .05127 & 4.1680 & 4.3707 \\
\hline
\end{tabular}

Table 7. Descriptives for perceived QoS differences between groups with the News app.

\begin{tabular}{lrlllll}
\hline $\begin{array}{l}\text { Connect } \\
\text { Speed }\end{array}$ & $\mathrm{N}$ & $\begin{array}{l}\text { News app } \\
\text { QoS mean }\end{array}$ & SD & SE & $\begin{array}{l}95 \% \text { CI } \\
\text { lower bound }\end{array}$ & $\begin{array}{l}\text { 95\% CI } \\
\text { upper bound }\end{array}$ \\
\hline Low & 47 & 3.8174 & .63313 & .09235 & 3.6315 & 4.0033 \\
Medium & 47 & 4.4309 & .52828 & .07706 & 4.2757 & 4.5860 \\
High & 48 & 4.4427 & .52653 & .07600 & 4.2898 & 4.5956 \\
Total & 142 & 4.2318 & .63232 & .05306 & 4.1269 & 4.3367 \\
\hline
\end{tabular}

Table 8. Tests of between-subjects effect for the Banking app.

\begin{tabular}{|c|c|c|c|c|}
\hline Source & $\begin{array}{l}\text { Type III sum } \\
\text { of squares }\end{array}$ & df & $\begin{array}{l}\text { Mean } \\
\text { square }\end{array}$ & $\mathrm{F}$ \\
\hline corrected model & 8.187 & 10 & .819 & $2.885^{* * *}$ \\
\hline Intercept & 20.309 & 1 & 20.309 & $71.555 * * *$ \\
\hline Gender & .804 & 1 & .804 & $2.832 *$ \\
\hline Prior GSM usage & .289 & 1 & .289 & 2.582 \\
\hline Prior SMS usage & .199 & 1 & .199 & $4.966^{* * *}$ \\
\hline Prior MMS usage & .03585 & 1 & .03585 & .126 \\
\hline Prior WAP usage & .009477 & 1 & .009477 & .033 \\
\hline Prior GPRS usage & 1.204 & 1 & 1.204 & $4.243 * *$ \\
\hline Prior mobile email usage & .06536 & 1 & .06536 & .230 \\
\hline Prior PDA usage & .733 & 1 & .733 & \\
\hline Bandwidth & 2.819 & 2 & 1.410 & \\
\hline Error & 36.896 & 130 & .284 & \\
\hline Total & 2795.563 & 141 & & \\
\hline Corrected total & 45.083 & 140 & & \\
\hline
\end{tabular}

${ }^{*} \mathrm{p}<.10,{ }^{* *} \mathrm{p}<.05,{ }^{* * *} \mathrm{p}<.01$ 
Table 9. Tests of between-subjects effect for the Email app.

\begin{tabular}{lcccc}
\hline Source & $\begin{array}{l}\text { Type III sum } \\
\text { of squares }\end{array}$ & df & $\begin{array}{l}\text { Mean } \\
\text { square }\end{array}$ & F \\
\hline Corrected model & 6.673 & 10 & .667 & $1.890^{*}$ \\
Intercept & 16.373 & 1 & 16.373 & $46.364^{* *}$ \\
Gender & .007569 & 1 & .007569 & .021 \\
Prior GSM usage & .03406 & 1 & .03406 & .010 \\
Prior SMS usage & .349 & 1 & .349 & .988 \\
Prior MMS usage & .0445 & 1 & .0445 & .126 \\
Prior WAP usage & .01735 & 1 & .01735 & .049 \\
Prior GPRS usage & 1.789 & 1 & 1.789 & $5.067^{* *}$ \\
Prior mobile email usage & .475 & 1 & .475 & 1.344 \\
Prior PDA usage & .02993 & 1 & .02993 & .085 \\
Bandwidth & 2.110 & 2 & 1.055 & $2.988^{*}$ \\
Error & 45.908 & 130 & .353 & \\
Total & 2620.688 & 141 & & \\
Corrected total & 52.581 & 140 & & \\
\hline
\end{tabular}

${ }^{*} \mathrm{p}<.10,{ }^{* *} \mathrm{p}<.05,{ }^{* * *} \mathrm{p}<.01$

Table 10. Tests of between-subjects effect with the News app.

\begin{tabular}{lcrcc}
\hline Source & $\begin{array}{l}\text { Type III sum } \\
\text { of squares }\end{array}$ & df & $\begin{array}{l}\text { Mean } \\
\text { square }\end{array}$ & $\mathrm{F}$ \\
\hline Corrected model & 16.258 & 10 & 1.626 & $5.575^{* * *}$ \\
Intercept & 11.145 & 1 & 11.145 & $38.220^{* * *}$ \\
Gender & .350 & 1 & .350 & 1.199 \\
Prior GSM usage & .678 & 1 & .678 & 2.325 \\
Prior SMS usage & .122 & 1 & .122 & .417 \\
Prior MMS usage & .00005279 & 1 & .00005279 & .000 \\
Prior WAP usage & .476 & 1 & .476 & 1.631 \\
Prior GPRS usage & .04277 & 1 & .04277 & .147 \\
Prior mobile email usage & .006823 & 1 &. .06823 & .023 \\
Prior PDA usage & .867 & 1 & .867 & $2.975^{*}$ \\
Bandwidth & 13.000 & 2 & 6.500 & $22.291^{* * *}$ \\
Error & 37.907 & 130 & .292 & \\
Total & 2591.778 & 141 & & \\
Corrected total & 54.165 & 140 & & \\
\hline
\end{tabular}

${ }^{*} \mathrm{p}<.10,{ }^{* *} \mathrm{p}<.05,{ }^{* * *} \mathrm{p}<.01$ 